\title{
On Delta and Nabla Caputo Fractional Differences and Dual Identities
}

\author{
Thabet Abdeljawad ${ }^{1,2}$ \\ ${ }^{1}$ Department of Mathematics and Physical Sciences, Prince Sultan University, P.O. Box 66833, Riyadh 11586, Saudi Arabia \\ ${ }^{2}$ Department of Mathematics, Çankaya University, 06530 Ankara, Turkey
}

Correspondence should be addressed to Thabet Abdeljawad; tabdeljawad@psu.edu.sa

Received 13 January 2013; Accepted 14 June 2013

Academic Editor: Shurong Sun

Copyright (C) 2013 Thabet Abdeljawad. This is an open access article distributed under the Creative Commons Attribution License, which permits unrestricted use, distribution, and reproduction in any medium, provided the original work is properly cited.

\begin{abstract}
We investigate two types of dual identities for Caputo fractional differences. The first type relates nabla and delta type fractional sums and differences. The second type represented by the Q-operator relates left and right fractional sums and differences. Two types of Caputo fractional differences are introduced; one of them (dual one) is defined so that it obeys the investigated dual identities. The relation between Riemann and Caputo fractional differences is investigated, and the delta and nabla discrete MittagLeffler functions are confirmed by solving Caputo type linear fractional difference equations. A nabla integration by parts formula is obtained for Caputo fractional differences as well.
\end{abstract}

\section{Introduction}

During the last two decades, due to its widespread applications in different fields of science and engineering, fractional calculus has attracted the attention of many researchers [1-6].

Starting from the idea of discretizing the Cauchy integral formula, Miller and Ross [7] and Gray and Zhang [8] obtained discrete versions of left type fractional integrals and derivatives, called fractional sums and differences. After that, several authors started to deal with discrete fractional calculus [9-20], benefiting from time scales calculus originated in 1988 (see [21]).

In [9], the concept of Caputo fractional difference was introduced and investigated. In this paper we proceed deeply to investigate Caputo fractional differences under two kinds of dual identities. The first kind relates nabla and delta type Caputo fractional differences and the second one, represented by the Q-operator, relates left and right ones. Arbitrary order Riemann and Caputo fractional differences are related as well. By the help of the previously obtained results in $[19,20]$ an integration by parts formula for Caputo fractional differences is originated.

The paper is organized as follows. The remaining part of this section contains summary to some of the basic notations and definitions in delta and nabla calculus. Section 2 contains the definitions in the frame of delta and nabla fractional sums and differences in the Riemann sense. The third section contains some dual identities relating nabla and delta type fractional sums and differences in Riemann sense as previously investigated in [20]. In Section 4 Caputo fractional differences are given and related to the Riemann ones. In Section 5, slightly different modified (dual) Caputo fractional differences are introduced and investigated under some dual identities. Section 6 is devoted to the integration by parts for delta and nabla Caputo fractional differences. Finally, Section 7 contains Caputo type fractional dynamical equations where a nonhomogeneous nabla Caputo fractional difference equation is solved to obtain nabla discrete versions for Mittag-Leffler functions. For the case $\alpha=1$ we obtain the discrete nabla exponential function [21]. In addition to this, the $Q$-operator is used to relate left and right Caputo fractional differences in the nabla and delta case. The $Q$-dual identities obtained in this section expose the validity of the definition of delta and nabla right Caputo fractional differences.

For a natural number $n$, the fractional polynomial is defined by.

$$
t^{(n)}=\prod_{j=0}^{n-1}(t-j)=\frac{\Gamma(t+1)}{\Gamma(t+1-n)}
$$


where $\Gamma$ denotes the special gamma function and the product is zero when $t+1-j=0$ for some $j$. More generally, for arbitrary $\alpha$, define

$$
t^{(\alpha)}=\frac{\Gamma(t+1)}{\Gamma(t+1-\alpha)},
$$

with the convention that division at pole yields zero. Given that the forward and backward difference operators are defined by

$$
\Delta f(t)=f(t+1)-f(t), \quad \nabla f(t)=f(t)-f(t-1),
$$

respectively, we define iteratively the operators $\Delta^{m}=\Delta\left(\Delta^{m-1}\right)$ and $\nabla^{m}=\nabla\left(\nabla^{m-1}\right)$, where $m$ is a natural number.

Here are some properties of the factorial function.

Lemma 1 (see [10]). Assume that the following factorial functions are well defined:

(i) $\Delta t^{(\alpha)}=\alpha t^{(\alpha-1)}$;

(ii) $(t-\mu) t^{(\mu)}=t^{(\mu+1)}$, where $\mu \in \mathbb{R}$;

(iii) $\mu^{(\mu)}=\Gamma(\mu+1)$;

(iv) If $t \leq r$, then $t^{(\alpha)} \leq r^{(\alpha)}$ for any $\alpha>r$;

(v) If $0<\alpha<1$, then $t^{(\alpha v)} \geq\left(t^{(v)}\right)^{\alpha}$;

(vi) $t^{(\alpha+\beta)}=(t-\beta)^{(\alpha)} t^{(\beta)}$.

Also, for our purposes we list down the following two properties, the proofs of which are straightforward:

$$
\begin{gathered}
\nabla_{s}(s-t)^{(\alpha-1)}=(\alpha-1)(\rho(s)-t)^{(\alpha-2)} ; \\
\nabla_{t}(\rho(s)-t)^{(\alpha-1)}=-(\alpha-1)(\rho(s)-t)^{(\alpha-2)} .
\end{gathered}
$$

For the sake of the nabla fractional calculus we have the following definition.

Definition 2 (see [21-24]). (i) For a natural number $m$, the $m$ rising (ascending) factorial of $t$ is defined by

$$
t^{\bar{m}}=\prod_{k=0}^{m-1}(t+k), \quad t^{\overline{0}}=1
$$
by

(ii) For any real number the $\alpha$ rising function is defined

$$
t^{\bar{\alpha}}=\frac{\Gamma(t+\alpha)}{\Gamma(t)}, \quad t \in \mathbb{R}-\{\ldots,-2,-1,0\}, 0^{\alpha}=0 .
$$

Regarding the rising factorial function we observe the following:

(i) $\nabla\left(t^{\bar{\alpha}}\right)=\alpha t^{\overline{\alpha-1}}$

(ii) $\left(t^{\bar{\alpha}}\right)=(t+\alpha-1)^{(\alpha)}$,

(iii) $\Delta_{t}(s-\rho(t))^{\bar{\alpha}}=-\alpha(s-\rho(t))^{\overline{\alpha-1}}$.
Notation

(i) For a real $\alpha>0$, we set $n=[\alpha]+1$, where $[\alpha]$ is the greatest integer less than $\alpha$.

(ii) For real numbers $a$ and $b$, we denote that $\mathbb{N}_{a}=\{a, a+$ $1, \ldots\}$ and ${ }_{b} \mathbb{N}=\{b, b-1, \ldots\}$.

(iii) For $n \in \mathbb{N}$ and real $a$, we denote that

$$
{ }_{\Theta} \Delta^{n} f(t) \triangleq(-1)^{n} \Delta^{n} f(t) \text {. }
$$

(iv) For $n \in \mathbb{N}$ and real $b$, we denote that

$$
\nabla_{\odot}^{n} f(t) \triangleq(-1)^{n} \nabla^{n} f(t)
$$

\section{Definitions and Essential Lemmas}

Definition 3. Let $\sigma(t)=t+1$ and $\rho(t)=t-1$ be the forward and backward jumping operators, respectively. Then we have the following.

(i) The (delta) left fractional sum of order $\alpha>0$ (starting from $a$ ) is defined by

$$
\Delta_{a}^{-\alpha} f(t)=\frac{1}{\Gamma(\alpha)} \sum_{s=a}^{t-\alpha}(t-\sigma(s))^{(\alpha-1)} f(s), \quad t \in \mathbb{N}_{a+\alpha} .
$$

(ii) The (delta) right fractional sum of order $\alpha>0$ (ending at $b$ ) is defined by

$$
\begin{aligned}
{ }_{b} \Delta^{-\alpha} f(t) & =\frac{1}{\Gamma(\alpha)} \sum_{s=t+\alpha}^{b}(s-\sigma(t))^{(\alpha-1)} f(s) \\
& =\frac{1}{\Gamma(\alpha)} \sum_{s=t+\alpha}^{b}(\rho(s)-t)^{(\alpha-1)} f(s), \quad t \in_{b-\alpha} \mathbb{N} .
\end{aligned}
$$

(iii) The (nabla) left fractional sum of order $\alpha>0$ (starting from $a$ ) is defined by

$$
\nabla_{a}^{-\alpha} f(t)=\frac{1}{\Gamma(\alpha)} \sum_{s=a+1}^{t}(t-\rho(s))^{\overline{\alpha-1}} f(s), \quad t \in \mathbb{N}_{a+1} \text {. }
$$

(iv) The (nabla) right fractional sum of order $\alpha>0$ (ending at $b$ ) is defined by

$$
\begin{aligned}
{ }_{b} \nabla^{-\alpha} f(t) & =\frac{1}{\Gamma(\alpha)} \sum_{s=t}^{b-1}(s-\rho(t))^{\overline{\alpha-1}} f(s) \\
& =\frac{1}{\Gamma(\alpha)} \sum_{s=t}^{b-1}(\sigma(s)-t)^{\overline{\alpha-1}} f(s), \quad t \in_{b-1} \mathbb{N} .
\end{aligned}
$$

Regarding the delta left fractional sum we observe the following.

(i) $\Delta_{a}^{-\alpha}$ maps functions defined on $\mathbb{N}_{a}$ to functions defined on $\mathbb{N}_{a+\alpha}$. 
(ii) $u(t)=\Delta_{a}^{-n} f(t), n \in \mathbb{N}$, satisfies the initial value problem

$$
\begin{gathered}
\Delta^{n} u(t)=f(t), \quad t \in N_{a}, \\
u(a+j-1)=0, \quad j=1,2, \ldots, n .
\end{gathered}
$$

(iii) The Cauchy function $(t-\sigma(s))^{(n-1)} /(n-1)$ ! vanishes at $s=t-(n-1), \ldots, t-1$.

Regarding the delta right fractional sum we observe the following.

(i) ${ }_{b} \Delta^{-\alpha}$ maps functions defined on ${ }_{b} \mathbb{N}$ to functions defined on ${ }_{b-\alpha} \mathbb{N}$.

(ii) $u(t)={ }_{b} \Delta^{-n} f(t), n \in \mathbb{N}$, satisfies the initial value problem

$$
\begin{gathered}
\nabla_{\ominus}^{n} u(t)=f(t), \quad t \in{ }_{b} N, \\
u(b-j+1)=0, \quad j=1,2, \ldots, n .
\end{gathered}
$$

(iii) the Cauchy function $(\rho(s)-t)^{(n-1)} /(n-1)$ ! vanishes at $s=t+1, t+2, \ldots, t+(n-1)$.

Regarding the nabla left fractional sum we observe the following.

(i) $\nabla_{a}^{-\alpha}$ maps functions defined on $\mathbb{N}_{a}$ to functions defined on $\mathbb{N}_{a}$.

(ii) $\nabla_{a}^{-n} f(t)$ satisfies the $n$th order discrete initial value problem

$\nabla^{n} y(t)=f(t), \quad \nabla^{i} y(a)=0, \quad i=0,1, \ldots, n-1$.

(iii) The Cauchy function $(t-\rho(s))^{\overline{n-1}} / \Gamma(n)$ satisfies $\nabla^{n} y(t)=0$.

Regarding the nabla right fractional sum we observe the following.

(i) ${ }_{b} \nabla^{-\alpha}$ maps functions defined on ${ }_{b} \mathbb{N}$ to functions defined on ${ }_{b} \mathbb{N}$.

(ii) ${ }_{b} \nabla^{-n} f(t)$ satisfies the $n$th order discrete initial value problem

${ }_{\ominus} \Delta^{n} y(t)=f(t), \quad{ }_{\ominus} \Delta^{i} y(b)=0, \quad i=0,1, \ldots, n-1$.

The proof can be done inductively. Namely, assuming it is true for $n$, we have

$$
{ }_{\ominus} \Delta^{n+1}{ }_{b} \nabla^{-(n+1)} f(t)={ }_{\ominus} \Delta^{n}\left[-\Delta_{b} \nabla^{-(n+1)} f(t)\right] .
$$

By the help of (9), it follows that

$$
{ }_{\ominus} \Delta^{n+1}{ }_{b} \nabla^{-(n+1)} f(t)={ }_{\ominus} \Delta^{n}{ }_{b} \nabla^{-n} f(t)=f(t) .
$$

The other part is clear by using the convention that $\sum_{k=s+1}^{s}=$ 0 . (iii) The Cauchy function $(s-\rho(t))^{\overline{n-1}} / \Gamma(n)$ satisfies ${ }_{\ominus} \Delta^{n} y(t)=0$.

Definition 4. (i) The (delta) left fractional difference [7] of order $\alpha>0$ (starting from $a$ ) is defined by

$$
\begin{aligned}
\Delta_{a}^{\alpha} f(t)= & \Delta^{n} \Delta_{a}^{-(n-\alpha)} f(t)=\frac{\Delta^{n}}{\Gamma(n-\alpha)} \\
& \times \sum_{s=a}^{t-(n-\alpha)}(t-\sigma(s))^{(n-\alpha-1)} f(s), \quad t \in \mathbb{N}_{a+(n-\alpha)} .
\end{aligned}
$$

(ii) The (delta) right fractional difference [15] of order $\alpha>$ 0 (ending at $b$ ) is defined by

$$
\begin{aligned}
{ }_{b} \Delta^{\alpha} f(t)= & \nabla_{\odot b}^{n} \Delta^{-(n-\alpha)} f(t)=\frac{(-1)^{n} \nabla^{n}}{\Gamma(n-\alpha)} \\
& \times \sum_{s=t+(n-\alpha)}^{b}(s-\sigma(t))^{(n-\alpha-1)} f(s), \quad t \in_{b-(n-\alpha)} \mathbb{N} .
\end{aligned}
$$

(iii) The (nabla) left fractional difference of order $\alpha>0$ (starting from $a$ ) is defined by

$$
\begin{aligned}
\nabla_{a}^{\alpha} f(t)= & \nabla^{n} \nabla_{a}^{-(n-\alpha)} f(t)=\frac{\nabla^{n}}{\Gamma(n-\alpha)} \\
& \times \sum_{s=a+1}^{t}(t-\rho(s))^{\overline{n-\alpha-1}} f(s), \quad t \in \mathbb{N}_{a+1} .
\end{aligned}
$$

(iv) The (nabla) right fractional difference of order $\alpha>0$ (ending at $b$ ) is defined by

$$
\begin{aligned}
{ }_{b} \nabla^{\alpha} f(t) & ={ }_{\Theta} \Delta^{n}{ }_{b} \nabla^{-(n-\alpha)} f(t) \\
& =\frac{(-1)^{n} \Delta^{n}}{\Gamma(n-\alpha)} \sum_{s=t}^{b-1}(s-\rho(t))^{\overline{n-\alpha-1}} f(s), \quad t \in{ }_{b-1} \mathbb{N} .
\end{aligned}
$$

Regarding the domains of the fractional type differences we observe the following.

(i) The delta left fractional difference $\Delta_{a}^{\alpha}$ maps functions defined on $\mathbb{N}_{a}$ to functions defined on $\mathbb{N}_{a+(n-\alpha)}$.

(ii) The delta right fractional difference ${ }_{b} \Delta^{\alpha}$ maps functions defined on ${ }_{b} \mathbb{N}$ to functions defined on ${ }_{b-(n-\alpha)} \mathbb{N}$.

(iii) The nabla left fractional difference $\nabla_{a}^{\alpha}$ maps functions defined on $\mathbb{N}_{a}$ to functions defined on $\mathbb{N}_{a+n}$ (on $\mathbb{N}_{a}$ if we think $f$ defined at some points before $a$ ).

(iv) The nabla right fractional difference ${ }_{b} \nabla^{\alpha}$ maps functions defined on ${ }_{b} \mathbb{N}$ to functions defined on ${ }_{b-n} \mathbb{N}$ (on ${ }_{b} \mathbb{N}$ if we think $f$ defined at some points after $b$ ).

Lemma 5 (see [10]). For any $\alpha>0$, the following equality holds:

$$
\Delta_{a}^{-\alpha} \Delta f(t)=\Delta \Delta_{a}^{-\alpha} f(t)-\frac{(t-a)^{\overline{\alpha-1}}}{\Gamma(\alpha)} f(a) .
$$


Lemma 6 (see [15]). For any $\alpha>0$, the following equality holds:

$$
{ }_{b} \Delta^{-\alpha} \nabla_{\odot} f(t)=\nabla_{\odot b} \Delta^{-\alpha} f(t)-\frac{(b-t)^{\overline{\alpha-1}}}{\Gamma(\alpha)} f(b) .
$$

Lemma 7 (see [25]). For any $\alpha>0$, the following equality holds:

$$
\nabla_{a+1}^{-\alpha} \nabla f(t)=\nabla \nabla_{a}^{-\alpha} f(t)-\frac{(t-a+1)^{\overline{\alpha-1}}}{\Gamma(\alpha)} f(a) .
$$

The result of Lemma 7 was obtained in [25] by applying the nabla left fractional sum starting from $a$ not from $a+1$. Next will provide the version of Lemma 7 by applying the definition in this paper. Actually, the nabla fractional sums defined in this paper and those in [25] are related. For more details we refer to [19].

Lemma 8 (see $[19,20]$ ). For any $\alpha>0$, the following equality holds:

$$
\nabla_{a}^{-\alpha} \nabla f(t)=\nabla \nabla_{a}^{-\alpha} f(t)-\frac{(t-a)^{\overline{\alpha-1}}}{\Gamma(\alpha)} f(a) .
$$

Remark 9 (see $[19,20])$. Let $\alpha>0$ and $n=[\alpha]+1$. Then, by the help of Lemma 8 we have

$$
\nabla \nabla_{a}^{\alpha} f(t)=\nabla \nabla^{n}\left(\nabla_{a}^{-(n-\alpha)} f(t)\right)=\nabla^{n}\left(\nabla \nabla_{a}^{-(n-\alpha)} f(t)\right)
$$

or

$$
\nabla \nabla_{a}^{\alpha} f(t)=\nabla^{n}\left[\nabla_{a}^{-(n-\alpha)} \nabla f(t)+\frac{(t-a)^{\overline{n-\alpha-1}}}{\Gamma(n-\alpha)} f(a)\right] .
$$

Then, using the identity

$$
\nabla^{n} \frac{(t-a)^{\overline{n-\alpha-1}}}{\Gamma(n-\alpha)}=\frac{(t-a)^{\overline{-\alpha-1}}}{\Gamma(-\alpha)}
$$

we infer that (29) is valid for any real $\alpha$.

By the help of Lemma 8 , Remark 9 , and the identity $\nabla(t-$ $a)^{\overline{\alpha-1}}=(\alpha-1)(t-a)^{\overline{\alpha-2}}$, we arrive inductively at the following generalization.

Theorem 10 (see $[19,20])$. For any real number $\alpha$ and any positive integer $p$, the following equality holds:

$$
\nabla_{a}^{-\alpha} \nabla^{p} f(t)=\nabla^{p} \nabla_{a}^{-\alpha} f(t)-\sum_{k=0}^{p-1} \frac{(t-a)^{\overline{\alpha-p+k}}}{\Gamma(\alpha+k-p+1)} \nabla^{k} f(a),
$$

where $f$ is defined on $\mathbb{N}_{a}$ and some points before $a$.

Lemma 11 (see $[19,20])$. For any $\alpha>0$, the following equality holds:

$$
{ }_{b} \nabla^{-\alpha}{ }_{\Theta} \Delta f(t)={ }_{{ } \Delta}{ }_{b} \nabla^{-\alpha} f(t)-\frac{(b-t)^{\overline{\alpha-1}}}{\Gamma(\alpha)} f(b) .
$$

Remark 12 (see $[19,20])$. Let $\alpha>0$ and $n=[\alpha]+1$. Then, by the help of Lemma 11 we can have

$$
\begin{aligned}
{ }_{{ } \Delta}{ }_{b} \nabla^{\alpha} f(t) & ={ }_{\ominus} \Delta{ }_{\ominus} \Delta^{n}\left({ }_{b} \nabla^{-(n-\alpha)} f(t)\right) \\
& ={ }_{\Theta} \Delta^{n}\left({ }_{{ }} \Delta_{b} \nabla^{-(n-\alpha)} f(t)\right)
\end{aligned}
$$

or

$$
\begin{aligned}
{ }_{\Theta} \Delta_{b} \nabla^{\alpha} f(t) \\
\quad{ }_{{ }_{\Theta}} \Delta^{n}\left[{ }_{b} \nabla^{-(n-\alpha)}{ }_{\Theta} \Delta f(t)+\frac{(b-t)^{\overline{n-\alpha-1}}}{\Gamma(n-\alpha)} f(b)\right] .
\end{aligned}
$$

Then, using the identity

$$
{ }_{\ominus} \Delta^{n} \frac{(b-t)^{\overline{n-\alpha-1}}}{\Gamma(n-\alpha)}=\frac{(b-t)^{\overline{-\alpha-1}}}{\Gamma(-\alpha)}
$$

we infer that (34) is valid for any real $\alpha$.

By the help of Lemma 11, Remark 12, and the identity $\Delta(b-t)^{\overline{\alpha-1}}=-(\alpha-1)(b-t)^{\overline{\alpha-2}}$, if we follow inductively we arrive at the following generalization.

Theorem 13 (see $[19,20])$. For any real number $\alpha$ and any positive integer $p$, the following equality holds:

$$
\begin{aligned}
{ }_{b} \nabla^{-\alpha}{ }_{\Theta} \Delta^{p} f(t) \\
\quad={ }_{\Theta} \Delta^{p}{ }_{b} \nabla^{-\alpha} f(t)-\sum_{k=0}^{p-1} \frac{(b-t)^{\overline{\alpha-p+k}}}{\Gamma(\alpha+k-p+1)}{ }^{{ }^{k}} \Delta^{k} f(b),
\end{aligned}
$$

where $f$ is defined on ${ }_{b} N$ and some points after $b$.

\section{Dual Identities for Fractional Sums and Riemann Fractional Differences}

The dual relations for left fractional sums and differences were investigated in [12]. Indeed, the following two lemmas are dual relations between the delta left fractional sums (differences) and the nabla left fractional sums (differences).

Lemma 14 (see [12]). Let $0 \leq n-1<\alpha \leq n$ and let $y(t)$ be defined on $\mathbb{N}_{a}$. Then the following statements are valid.
(i) $\left(\Delta_{a}^{\alpha}\right) y(t-\alpha)=\nabla_{a}^{\alpha} y(t)$ for $t \in \mathbb{N}_{n+a}$.
(ii) $\left(\Delta_{a}^{-\alpha}\right) y(t+\alpha)=\nabla_{a}^{-\alpha} y(t)$ for $t \in \mathbb{N}_{a}$.

Lemma 15 (see [12]). Let $0 \leq n-1<\alpha \leq n$ and let $y(t)$ be defined on $\mathbb{N}_{\alpha-n}$. Then the following statements are valid.
(i) $\Delta_{\alpha-n}^{\alpha} y(t)=\left(\nabla_{\alpha-n}^{\alpha} y\right)(t+\alpha)$ for $t \in \mathbb{N}_{-n}$
(ii) $\Delta_{\alpha-n}^{-(n-\alpha)} y(t)=\left(\nabla_{\alpha-n}^{-(n-\alpha)} y\right)(t-n+\alpha)$ for $t \in \mathbb{N}_{0}$.

We remind the reader that the previous two dual lemmas for left fractional sums and differences were obtained when the nabla left fractional sum was defined by

$$
\nabla_{a}^{-\alpha} f(t)=\frac{1}{\Gamma(\alpha)} \sum_{s=a}^{t}(t-\rho(s))^{\overline{\alpha-1}} f(s), \quad t \in \mathbb{N}_{a} .
$$


Now, in analogous to Lemmas 14 and 15, for the right fractional summations and differences the author in [20] obtained:

Lemma 16. Let $y(t)$ be defined on ${ }_{b+1} \mathbb{N}$. Then the following statements are valid.

(i) $\left({ }_{b} \Delta^{\alpha}\right) y(t+\alpha)={ }_{b+1} \nabla^{\alpha} y(t)$ for $t \in{ }_{b-n} \mathbb{N}$.

(ii) $\left({ }_{b} \Delta^{-\alpha}\right) y(t-\alpha)={ }_{b+1} \nabla^{-\alpha} y(t)$ for $t \in{ }_{b} \mathbb{N}$.

Lemma 17 (see [20]). Let $0 \leq n-1<\alpha \leq n$ and let $y(t)$ be defined on ${ }_{n-\alpha} \mathbb{N}$. Then the following statements are valid.

(i) ${ }_{n-\alpha} \Delta^{\alpha} y(t)={ }_{n-\alpha+1} \nabla^{\alpha} y(t-\alpha), t \in{ }_{n} \mathbb{N}$

(ii) ${ }_{n-\alpha} \Delta^{-(n-\alpha)} y(t)={ }_{n-\alpha+1} \nabla^{-(n-\alpha)} y(t+n-\alpha), t \in{ }_{0} \mathbb{N}$.

Proof. We prove (i), the proof of (ii) is similar. By the definition of right nabla difference we have

$$
\begin{aligned}
{ }_{n-\alpha+1} & \nabla^{\alpha} y(t-\alpha) \\
& ={ }_{a} \Delta^{n} \frac{1}{\Gamma(n-\alpha)} \sum_{s=t-\alpha}^{n-\alpha}(s-\rho(t-\alpha))^{\overline{n-\alpha-1}} y(s) \\
& ={ }_{a} \Delta^{n} \frac{1}{\Gamma(n-\alpha)} \sum_{s=t-\alpha}^{n-\alpha}(s-\rho(t-\alpha))^{\overline{n-\alpha-1}} y(s) \\
& =\nabla_{b}^{n} \frac{1}{\Gamma(n-\alpha)} \sum_{s=t+n-\alpha}^{n-\alpha}(s-\rho(t+n-\alpha))^{\frac{1-\alpha-1}{n-\alpha}} y(s) .
\end{aligned}
$$

By using (8) it follows that

$$
\begin{aligned}
{ }_{n-\alpha+1} & \nabla^{\alpha} y(t-\alpha) \\
& =\nabla_{b}^{n} \frac{1}{\Gamma(n-\alpha)} \sum_{s=t+n-\alpha}^{n-\alpha}(s-\sigma(t))^{(n-\alpha-1)} y(s) \\
& ={ }_{n-\alpha} \Delta^{\alpha} y(t) .
\end{aligned}
$$

Note that the previous two dual lemmas for right fractional differences cannot be obtained if we apply the definition of the delta right fractional difference introduced in $[13,14]$.

Lemma 18 (see [15]). Let $\alpha>0, \mu>0$. Then,

$$
{ }_{b-\mu} \Delta^{-\alpha}(b-t)^{(\mu)}=\frac{\Gamma(\mu+1)}{\Gamma(\mu+\alpha+1)}(b-t)^{(\mu+\alpha)} .
$$

The following commutative property for delta right fractional sums is Theorem 9 in [15].

Theorem 19. Let $\alpha>0, \mu>0$. Then, for all $t$ such that $t \equiv$ $b-(\mu+\alpha)(\bmod 1)$, one has

$$
{ }_{b} \Delta^{-\alpha}\left[{ }_{b} \Delta^{-\mu} f(t)\right]={ }_{b} \Delta^{-(\mu+\alpha)} f(t)={ }_{b} \Delta^{-\mu}\left[{ }_{b} \Delta^{-\alpha} f(t)\right],
$$

where $f$ is defined on ${ }_{b} N$.
Proposition 20 (see [20]). Let $f$ be a real valued function defined on ${ }_{b} \mathbb{N}$, and let $\alpha, \beta>0$. Then

$$
{ }_{b} \nabla^{-\alpha}\left[{ }_{b} \nabla^{-\beta} f(t)\right]={ }_{b} \nabla^{-(\alpha+\beta)} f(t)={ }_{b} \nabla^{-\beta}\left[{ }_{b} \nabla^{-\alpha} f(t)\right] .
$$

Proof. The proof follows by applying Lemma 16(ii) and Theorem 19 aforementioned. Indeed,

$$
\begin{aligned}
{ }_{b} \nabla^{-\alpha} & {\left[{ }_{b} \nabla^{-\beta} f(t)\right] } \\
& ={ }_{b} \nabla^{-\alpha}{ }_{b-1} \Delta^{-\beta} f(t-\beta) \\
& ={ }_{b-1} \Delta^{-\alpha}{ }_{b-1} \Delta^{-\beta} f(t-(\alpha+\beta)) \\
& ={ }_{b-1} \Delta^{-(\alpha+\beta)} f(t-(\alpha+\mu))={ }_{b} \nabla^{-(\alpha+\beta)} y(t) .
\end{aligned}
$$

The following power rule for nabla right fractional differences plays an important rule.

Proposition 21 (see $[19,20])$. Let $\alpha>0, \mu>-1$. Then, for $t \in{ }_{b} \mathbb{N}$, one has

$$
{ }_{b} \nabla^{-\alpha}(b-t)^{\bar{\mu}}=\frac{\Gamma(\mu+1)}{\Gamma(\alpha+\mu+1)}(b-t)^{\overline{\alpha+\mu}} .
$$

Proof. By the dual formula (ii) of Lemma 16, we have

$$
\begin{aligned}
& { }_{b} \nabla^{-\alpha}(b-t)^{\bar{\mu}} \\
& \quad=\left.{ }_{b-1} \Delta^{-\alpha}(b-r)^{\bar{\mu}}\right|_{r=t-\alpha} \\
& \quad=\frac{1}{\Gamma(\alpha)} \sum_{s=t}^{b-1}(s-t+\alpha-1)^{(\alpha-1)}(b-s)^{\bar{\mu}} .
\end{aligned}
$$

Then by the identity $t^{\bar{\alpha}}=(t+\alpha-1)^{(\alpha-1)}$ and using the change of variable $r=s-\mu+1$, it follows that

$$
\begin{aligned}
& { }_{b} \nabla^{-\alpha}(b-t)^{\bar{\mu}} \\
& \quad=\frac{1}{\Gamma(\alpha)} \sum_{r=t-\mu+1}^{b-\mu}(r-\sigma(t-\alpha-\mu+1))^{(\alpha-1)}(b-r)^{\bar{\mu}} \\
& =\left.\left({ }_{b-\mu} \Delta^{-\alpha}(b-u)^{\bar{\mu}}\right)\right|_{u=-\alpha-\mu+1+t} .
\end{aligned}
$$

This by Lemma 18 leads to

$$
\begin{aligned}
{ }_{b} \nabla^{-\alpha} & (b-t)^{\bar{\mu}} \\
& =\frac{\Gamma(\mu+1)}{\Gamma(\alpha+\mu+1)}(b-t+\alpha+\mu-1)^{(\alpha+\mu)} \\
& =\frac{\Gamma(\mu+1)}{\Gamma(\alpha+\mu+1)}(b-t)^{\overline{\alpha+\mu}} .
\end{aligned}
$$


Similarly, for the nabla left fractional sum we can have the following power formula and exponent law.

Proposition 22 (see $[19,20])$. Let $\alpha>0, \mu>-1$. Then, for $t \in \mathbb{N}_{a}$, one has

$$
\nabla_{a}^{-\alpha}(t-a)^{\bar{\mu}}=\frac{\Gamma(\mu+1)}{\Gamma(\alpha+\mu+1)}(t-a)^{\overline{\alpha+\mu}}
$$

Proposition 23 (see $[19,20]$ ). Let $f$ be a real valued function defined on $\mathbb{N}_{a}$, and let $\alpha, \beta>0$. Then

$$
\nabla_{a}^{-\alpha}\left[\nabla_{a}^{-\beta} f(t)\right]=\nabla_{a}^{-(\alpha+\beta)} f(t)=\nabla_{a}^{-\beta}\left[\nabla_{a}^{-\alpha} f(t)\right] .
$$

Proof. The proof can be achieved as in Theorem 2.1 [12], by expressing the left hand side of (51), interchanging the order of summation and using the power formula (50). Alternatively, the proof can be done by following as in the proof of Proposition 20 with the help of the dual formula for left fractional sum in Lemma 14 after its arrangement according to our definitions.

\section{Caputo Fractional Differences}

In analogous to the usual fractional calculus we can formulate the following definition.

Definition 24. Let $\alpha>0, \alpha \notin \mathbb{N}$. Then,

(i) the delta $\alpha$-order Caputo left fractional difference [9] of a function $f$ defined on $\mathbb{N}_{a}$ is defined by

$$
\begin{aligned}
{ }^{C} \Delta_{a}^{\alpha} f(t) & \triangleq \Delta_{a}^{-(n-\alpha)} \Delta^{n} f(t) \\
& =\frac{1}{\Gamma(n-\alpha)} \sum_{s=a}^{t-(n-\alpha)}(t-\sigma(s))^{(n-\alpha-1)} \Delta_{s}^{n} f(s) .
\end{aligned}
$$

(ii) the delta $\alpha$-order Caputo right fractional difference [9] of a function $f$ defined on ${ }_{b} \mathbb{N}$ is defined by

$$
\begin{aligned}
{ }_{b}^{C} \Delta^{\alpha} f(t) & \triangleq{ }_{b} \Delta^{-(n-\alpha)} \nabla_{\ominus}^{n} f(t) \\
& =\frac{1}{\Gamma(n-\alpha)} \sum_{s=t+(n-\alpha)}^{b}(s-\sigma(t))^{(n-\alpha-1)} \nabla_{\ominus}^{n} f(s),
\end{aligned}
$$

where $n=[\alpha]+1$.

If $\alpha=n \in \mathbb{N}$, then

$$
{ }^{C} \Delta_{a}^{\alpha} f(t) \triangleq \Delta^{n} f(t), \quad{ }_{b}^{C} \Delta^{\alpha} f(t) \triangleq \nabla_{b}^{n} f(t) .
$$

(iii) The nabla $\alpha$-order Caputo left fractional difference of a function $f$ defined on $\mathbb{N}_{a}$ and some points before $a$ is defined by

$$
\begin{aligned}
{ }^{C} \nabla_{a}^{\alpha} f(t) & \triangleq \nabla_{a}^{-(n-\alpha)} \nabla^{n} f(t) \\
& =\frac{1}{\Gamma(n-\alpha)} \sum_{s=a+1}^{t-(n-\alpha)}(t-\rho(s))^{\overline{n-\alpha-1}} \nabla^{n} f(s) .
\end{aligned}
$$

(iv) The nabla $\alpha$-order Caputo right fractional difference of a function $f$ defined on ${ }_{b} \mathbb{N}$ and some points after $b$ is defined by

$$
\begin{aligned}
{ }_{b}^{C} \nabla^{\alpha} f(t) & \triangleq{ }_{b} \nabla^{-(n-\alpha)} a \Delta^{n} f(t) \\
& =\frac{1}{\Gamma(n-\alpha)} \sum_{s=t}^{b-1}(s-\rho(t))^{\overline{n-\alpha-1}}{ }_{\ominus} \Delta^{n} f(s) .
\end{aligned}
$$

If $\alpha=n \in \mathbb{N}$, then

$$
{ }^{C} \nabla_{a}^{\alpha} f(t) \triangleq \nabla^{n} f(t), \quad{ }_{b}^{C} \nabla^{\alpha} f(t) \triangleq{ }_{a} \Delta^{n} f(t) .
$$

It is clear that ${ }^{C} \Delta_{a}^{\alpha}$ maps functions defined on $\mathbb{N}_{a}$ to functions defined on $\mathbb{N}_{a+(n-\alpha)}$ and that ${ }_{b} \Delta^{\alpha}$ maps functions defined on $_{b} \mathbb{N}$ to functions defined on ${ }_{b-(n-\alpha)} \mathbb{N}$. Also, it is clear that the nabla left fractional difference $\nabla_{a}^{\alpha}$ maps functions defined on $\mathbb{N}_{a}$ to functions defined on $\mathbb{N}_{a+1-n}$, and the nabla right fractional difference ${ }_{b} \nabla^{\alpha}$ maps functions defined on ${ }_{b} \mathbb{N}$ to functions defined on ${ }_{b-1+n} \mathbb{N}$.

Riemann and Caputo delta fractional differences are related by the following theorem.

Theorem 25 (see [9]). For any $\alpha>0$, one has

$$
\begin{gathered}
{ }_{\Delta_{a}}^{\alpha} f(t)=\Delta_{a}^{\alpha} f(t)-\sum_{k=0}^{n-1} \frac{(t-a)^{(k-\alpha)}}{\Gamma(k-\alpha+1)} \Delta^{k} f(a), \\
{ }_{b}^{C} \Delta^{\alpha} f(t)={ }_{b} \Delta^{\alpha} f(t)-\sum_{k=0}^{n-1} \frac{(b-t)^{(k-\alpha)}}{\Gamma(k-\alpha+1)} \nabla_{\ominus}^{k} f(b) .
\end{gathered}
$$

In particular, when $0<\alpha<1$, one has

$$
\begin{aligned}
& { }^{C} \Delta_{a} f(t)=\Delta_{a}^{\alpha} f(t)-\frac{(t-a)^{(-\alpha)}}{\Gamma(1-\alpha)} f(a), \\
& { }_{b}^{C} \Delta f(t)={ }_{b} \Delta^{\alpha} f(t)-\frac{(b-t)^{(-\alpha)}}{\Gamma(1-\alpha)} f(b) .
\end{aligned}
$$

One can note that the Riemann and Caputo fractional differences, for $0<\alpha<1$, coincide when $f$ vanishes at the end points.

The following identity is useful to transform delta type Caputo fractional difference equations into fractional summations.

Proposition 26 (see [9]). Assume that $\alpha>0$ and $f$ is defined on suitable domains $\mathbb{N}_{a}$ and ${ }_{b} \mathbb{N}$. Then,

$$
\begin{aligned}
& \Delta_{a+(n-\alpha)}^{-\alpha}{ }^{C} \Delta_{a}^{\alpha} f(t)=f(t)-\sum_{k=0}^{n-1} \frac{(t-a)^{(k)}}{k !} \Delta^{k} f(a), \\
& b-(n-\alpha) \Delta_{b}^{-\alpha} \Delta^{\alpha} f(t)=f(t)-\sum_{k=0}^{n-1} \frac{(b-t)^{(k)}}{k !} \nabla_{\ominus}^{k} f(b) .
\end{aligned}
$$

In particular, if $0<\alpha \leq 1$, then

$$
\begin{aligned}
& \Delta_{a+(n-\alpha)}^{-\alpha}{ }^{C} \Delta_{a}^{\alpha} f(t)=f(t)-f(a), \\
& b-(n-\alpha) \Delta_{b}^{-\alpha}{ }_{b}^{\alpha} f(t)=f(t)-f(b) .
\end{aligned}
$$


Similar to what we have earlier, for the nabla fractional differences we obtain the following.

Theorem 27. For any $\alpha>0$, one has

$$
\begin{gathered}
{ }^{C} \nabla_{a}^{\alpha} f(t)=\nabla_{a}^{\alpha} f(t)-\sum_{k=0}^{n-1} \frac{(t-a)^{\overline{k-\alpha}}}{\Gamma(k-\alpha+1)} \nabla^{k} f(a), \\
{ }_{b}^{C} \nabla^{\alpha} f(t)={ }_{b} \nabla^{\alpha} f(t)-\sum_{k=0}^{n-1} \frac{(b-t)^{\overline{k-\alpha}}}{\Gamma(k-\alpha+1)} \theta^{k} f(b) .
\end{gathered}
$$

In particular, when $0<\alpha<1$, one has

$$
\begin{gathered}
{ }^{C} \nabla_{a}^{\alpha} f(t)=\nabla_{a}^{\alpha} f(t)-\frac{(t-a)^{\overline{-\alpha}}}{\Gamma(1-\alpha)} f(a), \\
{ }_{b}^{C} \nabla^{\alpha} f(t)={ }_{b} \nabla^{\alpha} f(t)-\frac{(b-t)^{-\alpha}}{\Gamma(1-\alpha)} f(b) .
\end{gathered}
$$

Proof. The proof follows by replacing $\alpha$ by $n-\alpha$ and $p$ by $n$ in Theorems 10 and 13, respectively.

One can see that the nabla Riemann and Caputo fractional differences, for $0<\alpha<1$, coincide when $f$ vanishes at the end points.

Proposition 28. Assume that $\alpha>0$ and $f$ is defined on suitable domains $\mathbb{N}_{a}$ and ${ }_{b} \mathbb{N}$. Then,

$$
\begin{gathered}
\nabla_{a}^{-\alpha}{ }^{C} \nabla_{a}^{\alpha} f(t)=f(t)-\sum_{k=0}^{n-1} \frac{(t-a)^{\bar{k}}}{k !} \nabla^{k} f(a), \\
{ }_{b} \nabla_{b}^{-\alpha} \nabla^{\alpha} f(t)=f(t)-\sum_{k=0}^{n-1} \frac{(b-t)^{\bar{k}}}{k !}{ }_{\ominus} \Delta^{k} f(b) .
\end{gathered}
$$

In particular, if $0<\alpha \leq 1$, then

$$
\begin{aligned}
& \nabla_{a}^{-\alpha C} \nabla_{a}^{\alpha} f(t)=f(t)-f(a), \\
& { }_{b} \nabla_{b}^{-\alpha C} \nabla^{\alpha} f(t)=f(t)-f(b) .
\end{aligned}
$$

Proof. The proof of (64) follows by the definition and applying Proposition 23 and (85) of Proposition 38. The proof of (65) follows by the definition and applying Proposition 20 and (87) of Proposition 39.

Using the definition and Propositions 22 and 21, we can find the nabla type Caputo fractional differences for certain power functions. For example, for $1 \neq \beta>0$ and $\alpha \geq 0$, we have

$$
\begin{aligned}
& { }_{\nabla_{a}^{C}}^{\alpha}(t-a)^{\overline{\beta-1}}=\frac{\Gamma(\beta)}{\Gamma(\beta-\alpha)}(t-a)^{\overline{\beta-\alpha-1}}, \\
& { }_{b}^{C} \nabla^{\alpha}(b-t)^{\overline{\beta-1}}=\frac{\Gamma(\beta)}{\Gamma(\beta-\alpha)}(b-t)^{\overline{\beta-\alpha-1}} .
\end{aligned}
$$

However,

$$
{ }^{C} \nabla_{a}^{\alpha} 1={ }_{b}^{C} \nabla^{\alpha} 1=0
$$

whereas

$$
\nabla_{a}^{\alpha} 1=\frac{(t-a)^{(-\alpha)}}{\Gamma(1-\alpha)}, \quad{ }_{b} \nabla^{\alpha} 1=\frac{(b-t)^{(-\alpha)}}{\Gamma(1-\alpha)} .
$$

In the previous formulae (67), we apply the convention that dividing over a pole leads to zero. Therefore, the fractional difference when $\beta-1=\alpha-j, j=1,2, \ldots, n$, is zero.

Remark 29. The results obtained in Theorem 25 and afterward agree with those in the usual continuous case (see [3] pages 91, 96).

\section{A Dual Nabla Caputo Fractional Difference}

In the previous section the nabla Caputo fractional difference is defined under the assumption that $f$ is known before $a$ in the left case and under the assumption that $f$ is known after $b$ in the right case. In this section we define other nabla Caputo fractional differences for which it is not necessary to request any information about $f$ before $a$ or after $b$. Since we will show that these Caputo fractional differences are the dual ones for the delta Caputo fractional differences; we call them dual nabla Caputo fractional differences.

Definition 30. Let $\alpha>0, n=[\alpha]+1, a(\alpha)=a+n-1$, and $b(\alpha)=b-n+1$. Then the dual nabla left and right Caputo fractional differences are defined by

$$
\begin{gathered}
{ }^{C} \nabla_{a(\alpha)}^{\alpha} f(t)=\nabla_{a(\alpha)}^{-(n-\alpha)} \nabla^{n} f(t), \quad t \in \mathbb{N}_{a+n}, \\
{ }_{b(\alpha)}^{C} \nabla^{\alpha} f(t)={ }_{b(\alpha)} \nabla^{-(n-\alpha)} \ominus \Delta^{n} f(t), \quad t \in_{b-n} \mathbb{N},
\end{gathered}
$$

respectively.

Notice that the Caputo and the dual Caputo differences coincide when $0<\alpha \leq 1$ and differ for higher order. That is, for $0<\alpha \leq 1$,

$$
{ }^{C} \nabla_{a(\alpha)}^{\alpha} f(t)={ }^{C} \nabla_{a}^{\alpha} f(t), \quad{ }_{b(\alpha)}^{C} \nabla^{\alpha} f(t)={ }_{b}^{C} \nabla^{\alpha} f(t) .
$$

The following proposition states a dual relation between left delta Caputo fractional differences and left nabla (dual) Caputo fractional differences.

Proposition 31. For $\alpha>0, n=[\alpha]+1, a(\alpha)=a+n-1$, one has

$$
\left({ }^{C} \Delta_{a}^{\alpha} f\right)(t-\alpha)=\left({ }^{C} \nabla_{a(\alpha)}^{\alpha} f\right)(t), \quad t \in N_{a+n} .
$$


Proof. For $t \in N_{a+n}$, we have

$$
\begin{aligned}
& \left({ }^{C} \Delta_{a}^{\alpha} f\right)(t-\alpha) \\
& \quad=\frac{1}{\Gamma(n-\alpha)} \sum_{s=a}^{t-n}(t-\alpha-\sigma(s))^{(n-\alpha-1)} \Delta^{n} f(s) \\
& \quad=\frac{1}{\Gamma(n-\alpha)} \sum_{s=a}^{t-n}(t-\alpha-\sigma(s))^{(n-\alpha-1)} \nabla^{n} f(s+n) \\
& =\frac{1}{\Gamma(n-\alpha)} \sum_{r=a+n}^{t}(t-\rho(r)+n-\alpha-2)^{(n-\alpha-1)} \nabla^{n} f(r) \\
& \quad=\frac{1}{\Gamma(n-\alpha)} \sum_{r=a+n}^{t}(t-\rho(r))^{\frac{1-\alpha-1}{n}} \nabla^{n} f(r) \\
& =\left({ }^{C} \nabla_{a(\alpha)}^{\alpha} f\right)(t) .
\end{aligned}
$$

Analogously, the following proposition relates right delta Caputo fractional differences and right nabla (dual) Caputo fractional differences.

Proposition 32. For $\alpha>0, n=[\alpha]+1, b(\alpha)=b-n+1$, one has

$$
\left({ }_{b}^{C} \Delta^{\alpha} f\right)(t+\alpha)=\left({ }_{b(\alpha)}^{C} \nabla^{\alpha} f\right)(t), \quad t \in{ }_{b-n} \mathbb{N} .
$$

The following theorem modifies Theorem 10 when $f$ is only defined at $\mathbb{N}_{a}$.

Theorem 33. For any real number $\alpha$ and any positive integer $p$, the following equality holds:

$$
\begin{array}{r}
\nabla_{a+p-1}^{-\alpha} \nabla^{p} f(t)=\nabla^{p} \nabla_{a+p-1}^{-\alpha} f(t) \\
-\sum_{k=0}^{p-1} \frac{(t-(a+p-1))^{\overline{\alpha-p+k}}}{\Gamma(\alpha+k-p+1)} \\
\quad \times \nabla^{k} f(a+p-1),
\end{array}
$$

where $f$ is defined on only $\mathbb{N}_{a}$.

The proof follows by applying Remark 9 inductively.

Similarly, in the right case we have the following.

Theorem 34. For any real number $\alpha$ and any positive integer $p$, the following equality holds:

$$
\begin{aligned}
&{ }_{b-p+1} \nabla^{-\alpha}{ }_{\Theta} \Delta^{p} f(t) \\
&={ }_{\Theta} \Delta^{p}{ }_{b-p+1} \nabla^{-\alpha} f(t) \\
&-\sum_{k=0}^{p-1} \frac{(b-p+1-t)^{\overline{\alpha-p+k}}}{\Gamma(\alpha+k-p+1)}{ }_{\ominus}^{k} f(b-p+1),
\end{aligned}
$$

where $f$ is defined on ${ }_{b} \mathrm{~N}$ only.
Now by using the modified Theorems 33 and 34 we have the following.

Theorem 35. For any $\alpha>0$, one has

$$
\begin{gathered}
{ }^{C} \nabla_{a(\alpha)}^{\alpha} f(t)=\nabla_{a(\alpha)}^{\alpha} f(t)-\sum_{k=0}^{n-1} \frac{(t-a(\alpha))^{\overline{k-\alpha}}}{\Gamma(k-\alpha+1)} \nabla^{k} f(a(\alpha)), \\
{ }_{b(\alpha)}^{C} \nabla^{\alpha} f(t)={ }_{b(\alpha)} \nabla^{\alpha} f(t)-\sum_{k=0}^{n-1} \frac{(b(\alpha)-t)^{\overline{k-\alpha}}}{\Gamma(k-\alpha+1)} \theta^{k} f(b(\alpha)) .
\end{gathered}
$$

In particular, when $0<\alpha<1$, then $a(\alpha)=a$, and $b(\alpha)=b$, and hence one has

$$
\begin{gathered}
{ }^{C} \nabla_{a}^{\alpha} f(t)=\nabla_{a}^{\alpha} f(t)-\frac{(t-a)^{\overline{-\alpha}}}{\Gamma(1-\alpha)} f(a), \\
{ }_{b}^{C} \nabla^{\alpha} f(t)={ }_{b} \nabla^{\alpha} f(t)-\frac{(b-t)^{-\alpha}}{\Gamma(1-\alpha)} f(b) .
\end{gathered}
$$

Also, by using the modified Theorems 33 and 34 we have the following.

Proposition 36. Assume that $\alpha>0$ and $f$ is defined on suitable domains $\mathbb{N}_{a}$ and ${ }_{b} \mathbb{N}$. Then,

$$
\begin{gathered}
\nabla_{a(\alpha)}^{-\alpha}{ }^{C} \nabla_{a(\alpha)}^{\alpha} f(t)=f(t)-\sum_{k=0}^{n-1} \frac{(t-a(\alpha))^{\bar{k}}}{k !} \nabla^{k} f(a(\alpha)), \\
b(\alpha) \nabla_{b(\alpha)}^{-\alpha C} \nabla^{\alpha} f(t)=f(t)-\sum_{k=0}^{n-1} \frac{(b(\alpha)-t)^{\bar{k}}}{k !}{ }_{\ominus} \Delta^{k} f(b(\alpha)) .
\end{gathered}
$$

In particular, if $0<\alpha \leq 1$, then $a(\alpha)=a$, and $b(\alpha)=b$, and hence,

$$
\begin{aligned}
& \nabla_{a}^{-\alpha C} \nabla_{a}^{\alpha} f(t)=f(t)-f(a), \\
& { }_{b} \nabla_{b}^{-\alpha} \nabla^{\alpha} f(t)=f(t)-f(b) .
\end{aligned}
$$

\section{Integration by Parts for Caputo Fractional Differences}

In this section we state the integration by parts formulas for nabla fractional sums and differences obtained in [19] then use the dual identities to obtain delta integration by part formulas.

Proposition 37 (see [19]). For $\alpha>0, a, b \in \mathbb{R}, f$ defined on $\mathbb{N}_{a}$ and $g$ defined on ${ }_{b} \mathbb{N}$, one has

$$
\sum_{s=a+1}^{b-1} g(s) \nabla_{a}^{-\alpha} f(s)=\sum_{s=a+1}^{b-1} f(s)_{b} \nabla^{-\alpha} g(s) .
$$


Proof. By the definition of the nabla left fractional sum we have

$$
\begin{aligned}
& \sum_{s=a+1}^{b-1} g(s) \nabla_{a}^{-\alpha} f(s) \\
& \quad=\frac{1}{\Gamma(\alpha)} \sum_{s=a+1}^{b-1} g(s) \sum_{r=a+1}^{s}(s-\rho(r))^{\overline{\alpha-1}} f(r) .
\end{aligned}
$$

If we interchange the order of summation we reach at (82).

By the help of Theorem 10, Proposition 23, (18), and that $\nabla_{a}^{-(n-\alpha)} f(a)=0$, the authors in [19] obtained the following left important tools which lead to a nabla integration by parts formula for fractional differences.

Proposition 38 (see [19]). For $\alpha>0$, and $f$ defined in $a$ suitable domain $\mathbb{N}_{a}$, one has

$$
\begin{gathered}
\nabla_{a}^{\alpha} \nabla_{a}^{-\alpha} f(t)=f(t), \\
\nabla_{a}^{-\alpha} \nabla_{a}^{\alpha} f(t)=f(t), \quad \text { when } \alpha \notin \mathbb{N}, \\
\nabla_{a}^{-\alpha} \nabla_{a}^{\alpha} f(t) \\
=f(t)-\sum_{k=0}^{n-1} \frac{(t-a)^{\bar{k}}}{k !} \nabla^{k} f(a), \quad \text { when } \alpha=n \in \mathbb{N} .
\end{gathered}
$$

By the help of Theorem 13, Proposition 20, (19), and that ${ }_{b} \nabla^{-(n-\alpha)} f(b)=0$, the authors also in [19] also obtained the following right important tool.

Proposition 39 (see [19]). For $\alpha>0$, and $f$ defined in a suitable domain ${ }_{b} \mathbb{N}$, one has

$$
\begin{gathered}
{ }_{b} \nabla^{\alpha}{ }_{b} \nabla^{-\alpha} f(t)=f(t), \\
{ }_{b} \nabla^{-\alpha}{ }_{b} \nabla^{\alpha} f(t)=f(t), \quad \text { when } \alpha \notin \mathbb{N}, \\
{ }_{b} \nabla^{-\alpha}{ }_{b} \nabla^{\alpha} f(t) \\
=f(t)-\sum_{k=0}^{n-1} \frac{(b-t)^{\bar{k}}}{k !}{ }_{a} \Delta^{k} f(b), \quad \text { when } \alpha=n \in \mathbb{N} .
\end{gathered}
$$

Proposition 40 (see [19]). Let $\alpha>0$ be noninteger and $a, b \in$ $\mathbb{R}$ such that $a<b$ and $b \equiv a(\bmod 1)$. If $f$ is defined on ${ }_{b} N$ and $g$ is defined on $N_{a}$, then

$$
\sum_{s=a+1}^{b-1} f(s) \nabla_{a}^{\alpha} g(s)=\sum_{s=a+1}^{b-1} g(s){ }_{b} \nabla^{\alpha} f(s) .
$$

The proof was achieved by making use of Proposition 37 and the tools Propositions 38 and 39.

By the previous nabla integration by parts formulas and the dual identities in Lemma 14 adjusted to our definitions and Lemma 16, in [20] the following delta integration by parts formulas were obtained.
Proposition 41 (see [20]). Let $\alpha>0, a, b \in \mathbb{R}$ such that $a<b$ and $b \equiv a(\bmod 1)$. If $f$ is defined on $N_{a}$ and $g$ is defined on ${ }_{b} N$, then one has

$$
\begin{aligned}
& \sum_{s=a+1}^{b-1} g(s)\left(\Delta_{a+1}^{-\alpha} f\right)(s+\alpha) \\
& \quad=\sum_{s=a+1}^{b-1} f(s)_{b-1} \Delta^{-\alpha} g(s-\alpha) .
\end{aligned}
$$

Proposition 42 (see [20]). Let $\alpha>0$ be noninteger and assume that $b \equiv a(\bmod 1)$. If $f$ is defined on ${ }_{b} N$ and $g$ is defined on $N_{a}$, then

$$
\begin{aligned}
\sum_{s=a+1}^{b-1} f(s) \Delta_{a+1}^{\alpha} g(s-\alpha) & \\
& =\sum_{s=a+1}^{b-1} g(s)_{b-1} \Delta^{\alpha} f(s+\alpha) .
\end{aligned}
$$

Now, we proceed in this section to obtain nabla and delta integration by parts formulas for Caputo fractional differences.

Theorem 43. Let $0<\alpha<1$ and let $f, g$ be functions defined on $\mathbb{N}_{a} \cap{ }_{b} \mathbb{N}$ where $a \equiv b(\bmod 1)$. Then,

$$
\begin{aligned}
& \sum_{s=a+1}^{b-1} g(s){ }^{C} \nabla_{a}^{\alpha} f(s) \\
& \quad=\left.f(s)_{b} \nabla^{-(1-\alpha)} g(s)\right|_{a} ^{b-1}+\sum_{s=a}^{b-2} f(s)_{b} \nabla^{\alpha} g(s),
\end{aligned}
$$

where clearly ${ }_{b} \nabla^{-(1-\alpha)} g(b-1)=g(b-1)$.

Proof. From the definition of Caputo fractional difference and Proposition 37 we have

$$
\begin{aligned}
& \sum_{s=a+1}^{b-1} g(s){ }^{C} \nabla_{a}^{\alpha} f(s) \\
& \quad=\sum_{s=a+1}^{b-1} g(s) \nabla_{a}^{-(1-\alpha)} \nabla f(s)=\sum_{s=a+1}^{b-1} \nabla f(s)_{b} \nabla^{-(1-\alpha)} g(s) .
\end{aligned}
$$

By integration by parts from difference calculus, $\nabla f(s)=$ $\Delta f(s-1)$, and the definition of nabla right fractional difference, we reach at

$$
\begin{aligned}
& \sum_{s=a+1}^{b-1} g(s){ }^{C} \nabla_{a}^{\alpha} f(s) \\
& \quad=\left.f(s){ }_{b} \nabla^{-(1-\alpha)} g(s)\right|_{a} ^{b-1}+\sum_{s=a+1}^{b-1} f(s-1)\left({ }_{b} \nabla^{\alpha} g\right)(s-1),
\end{aligned}
$$

from which (91) follows. 
Theorem 44. Let $0<\alpha<1$ and let $f, g$ be functions defined on $\mathbb{N}_{a} \cap{ }_{b} \mathbb{N}$ where $a \equiv b(\bmod 1)$. Then,

$$
\begin{aligned}
\sum_{s=a+1}^{b+1} g(s) & { }^{C} \Delta_{a}^{\alpha} f(s-\alpha) \\
= & \left.f(s)_{b-1} \Delta^{-(1-\alpha)} g(s-(1-\alpha))\right|_{a} ^{b-1} \\
& +\sum_{a}^{b-2} f(s)_{b-1} \Delta^{\alpha} g(s+\alpha) .
\end{aligned}
$$

Proof. By the dual Caputo identity (73) in Proposition 31 and Theorem 43 we have

$$
\begin{aligned}
& \sum_{s=a+1}^{b+1} g(s){ }^{C} \Delta_{a}^{\alpha} f(s-\alpha) \\
& \quad=\left.f(s)_{b} \nabla^{-(1-\alpha)}\right|_{a} ^{b-1}+\sum_{s=a}^{b-2} f(s)_{b} \nabla^{\alpha} g(s) .
\end{aligned}
$$

Then, (94) follows by Lemma 16 (i, ii).

\section{The Q-Operator and Fractional Difference Equations}

If $f(s)$ is defined on $N_{a} \cap{ }_{b} N$ and $a \equiv b(\bmod 1)$ then $(Q f)(s)=f(a+b-s)$. The $Q$-operator generates a dual identity by which the left type and the right type fractional sums and differences are related. Using the change of variable $u=a+b-s$, in [9], it was shown that

$$
\Delta_{a}^{-\alpha} Q f(t)=Q_{b} \Delta^{-\alpha} f(t)
$$

and, hence,

$$
{ }^{C} \Delta_{a}^{\alpha} Q f(t)=Q\left({ }_{b}^{C} \Delta^{\alpha} f\right)(t)
$$

The proof of (97) follows by the definition, (96), and by noting that

$$
-Q \nabla f(t)=\Delta Q f(t) .
$$

Similarly, in the nabla case we have

$$
\nabla_{a}^{-\alpha} Q f(t)=Q_{b} \nabla^{-\alpha} f(t)
$$

and, hence,

$$
{ }^{C} \nabla_{a}^{\alpha} Q f(t)=Q\left({ }_{b}^{C} \nabla^{\alpha} f\right)(t) .
$$

The proof of (100) follows by the definition, (99), and that

$$
-Q \Delta f(t)=\nabla Q f(t) \text {. }
$$

The Q-dual identities (97) and (100) are still valid for the delta and nabla (Riemann) fractional differences, respectively. The proof is similar to the Caputo case aforementioned.

It is remarkable to mention that the Q-dual identity (97) can not be obtained if the definition of the delta right fractional difference introduced by Bastos et al. in [14] or by Atıcı and Şengül in [13] is used. Thus, the definition introduced in $[9,15]$ is more convenient. Analogously, the Qdual identity (100) indicates that the nabla right Riemann and Caputo fractional differences presented in this paper are also more convenient.

It is clear from the previous argument that the $Q$-operator agrees with its continuous counterpart when applied to left and right fractional Riemann integrals and the Caputo and Riemann derivatives. More generally, this discrete version of the $Q$-operator can be used to transform the discrete delaytype fractional functional difference dynamic equations to advanced ones. For details in the continuous counterparts see [26].

Example 45 (see [9]). Let $0<\alpha \leq 1, a=\alpha-1$ and consider the left Caputo nonhomogeneous fractional difference equation

$$
{ }^{C} \Delta_{a}^{\alpha} y(t)=\lambda y(t+\alpha-1)+f(t), \quad y(a)=a_{0}, \quad t \in \mathbb{N}_{0} .
$$

Note that the solution $y(t)$, if exists, is defined on $\mathbb{N}_{a}$, and, hence, ${ }^{C} \Delta_{a}^{\alpha} y(t)$ becomes defined on $\mathbb{N}_{a+(1-\alpha)}=\mathbb{N}_{0}$. Thus, if we apply $\Delta_{0}^{-\alpha}$ on (102) and then use the successive approximation the following delta type solution is obtained:

$$
y(t)=a_{0} E_{(\alpha)}(\lambda, t)+\sum_{s=0}^{t-\alpha} E_{(\alpha, \alpha)}(\lambda, t-\sigma(s)) f(s),
$$

where the delta type discrete Mittag-Leffler functions are defined as follows.

Definition 46 (delta discrete Mittag-Leffler, [9]). For $\lambda \in \mathbb{R}$ and $\alpha, \beta, z \in \mathbb{C}$ with $\operatorname{Re}(\alpha)>0$, the delta discrete (like) Mittag-Leffler functions are defined by

$$
\begin{aligned}
E_{(\alpha, \beta)}(\lambda, z) & \\
& =\sum_{k=0}^{\infty} \lambda^{k} \frac{(z+(k-1)(\alpha-1))^{(k \alpha)}(z+k(\alpha-1))^{(\beta-1)}}{\Gamma(\alpha k+\beta)} .
\end{aligned}
$$

For $\beta=1$, it is written that

$$
E_{(\alpha)}(\lambda, z) \triangleq E_{(\alpha, 1)}(\lambda, z)=\sum_{k=0}^{\infty} \lambda^{k} \frac{(z+(k-1)(\alpha-1))^{(k \alpha)}}{\Gamma(\alpha k+1)} .
$$

Next, we solve nabla Caputo type nonhomogeneous fractional difference equation to formulate nabla type discrete Mittag-Leffler functions. Those functions generalize the nabla discrete exponential functions. For details about delta and nabla type exponential functions on arbitrary time scales we refer the reader to ([21], pages 10,76$)$. 
Definition 47 (nabla discrete Mittag-Leffler). For $\lambda \in \mathbb{R}$, and $\alpha, \beta, z \in \mathbb{C}$ with $\operatorname{Re}(\alpha)>0$, the nabla discrete (like) MittagLeffler functions are defined by

$$
E_{\overline{\alpha, \beta}}(\lambda, z)=\sum_{k=0}^{\infty} \lambda^{k} \frac{z^{\overline{k \alpha+\beta-1}}}{\Gamma(\alpha k+\beta)} .
$$

For $\beta=1$, it is written that

$$
E_{\bar{\alpha}}(\lambda, z) \triangleq E_{\overline{\alpha, 1}}(\lambda, z)=\sum_{k=0}^{\infty} \lambda^{k} \frac{z^{\overline{k \alpha}}}{\Gamma(\alpha k+1)} .
$$

Example 48. Let $0<\alpha \leq 1, a \in \mathbb{R}$ and consider the nabla left Caputo nonhomogeneous fractional difference equation

$$
{ }^{C} \nabla_{a}^{\alpha} y(t)=\lambda y(t)+f(t), \quad y(a)=a_{0}, \quad t \in \mathbb{N}_{a} .
$$

If we apply $\nabla_{a}^{-\alpha}$ on (108), then by (66) we see that

$$
y(t)=a_{0}+\frac{\lambda}{\Gamma(\alpha)} \sum_{s=a+1}^{t}(t-\rho(s))^{\overline{\alpha-1}} y(s)+\nabla_{a}^{-\alpha} f(t) .
$$

To obtain an explicit solution, we apply the method of successive approximation. Set $y_{0}(t)=a_{0}$ and

$$
y_{m}(t)=a_{0}+\nabla_{a}^{-\alpha}\left[\lambda y_{m-1}(t)+f(t)\right], \quad m=1,2,3, \ldots .
$$

For $m=1$, we have by the power formula (50) that

$$
y_{1}(t)=a_{0}\left[1+\frac{\lambda(t-a)^{\bar{\alpha}}}{\Gamma(\alpha+1)}\right]+\nabla_{a}^{-\alpha} f(t) .
$$

For $m=2$, we also see by the help of Proposition 23 that

$$
\begin{aligned}
y_{2}(t)= & a_{0}+\lambda \nabla_{a}^{-\alpha}\left[a_{0}+\frac{(t-a)^{\bar{\alpha}}}{\Gamma(\alpha+1)}\right]+\nabla_{a}^{-\alpha} f(t)+\lambda \nabla_{a}^{-2 \alpha} f(t) \\
= & a_{0}\left[1+\frac{\lambda t^{(\alpha)}}{\Gamma(\alpha+1)}+\frac{\lambda^{2}(t-a)^{\overline{2 \alpha}}}{\Gamma(2 \alpha+1)}\right] \\
& +\nabla_{a}^{-\alpha} f(t)+\lambda \nabla_{a}^{-2 \alpha} f(t) .
\end{aligned}
$$

Proceeding inductively and making use of Proposition 23 and let $m \rightarrow \infty$ we obtain the solution

$$
\begin{aligned}
y(t)= & a_{0} \sum_{k=0}^{\infty} \frac{\lambda^{k}(t-a)^{\overline{k \alpha}}}{\Gamma(k \alpha+1)} \\
& +\sum_{k=1}^{\infty} \lambda^{k-1}\left(\nabla_{a}^{-k \alpha} f\right)(t) .
\end{aligned}
$$

Then,

$$
\begin{aligned}
y(t)= & a_{0} E_{\bar{\alpha}}(\lambda, t-a) \\
& +\sum_{k=0}^{\infty} \lambda^{k} \frac{1}{\Gamma(\alpha k+\alpha)} \sum_{s=a+1}^{t}(t-\rho(s))^{\overline{k \alpha+\alpha-1}} f(s) .
\end{aligned}
$$

Interchanging the order of sums in (114), we conclude that

$$
y(t)=a_{0} E_{\bar{\alpha}}(\lambda, t-a)+\sum_{s=a+1}^{t} \sum_{k=0}^{\infty} \lambda^{k} \frac{(t-\rho(s))^{\overline{k \alpha+\alpha-1}}}{\Gamma(\alpha k+\alpha)} f(s) .
$$

That is,

$$
y(t)=a_{0} E_{\bar{\alpha}}(\lambda, t-a)+\sum_{s=a+1}^{t} E_{\overline{\alpha, \alpha}}(\lambda, t-\rho(s)) f(s) .
$$

Remark 49. If we solve the nabla discrete fractional system (108) with $\alpha=1$ and $a_{0}=1$, we obtain the solution

$$
\begin{aligned}
y(t)= & \sum_{k=0}^{\infty} \lambda^{k} \frac{(t-a)^{\bar{k}}}{k !} \\
& +\sum_{s=a+1}^{t} \sum_{k=0}^{\infty} \lambda^{k} \frac{(t-\rho(s))^{\bar{k}}}{k !} f(s) .
\end{aligned}
$$

The first part of the solution is the nabla discrete exponential function $\widehat{e}_{\lambda}(t, a)$. For the sake of more comparisons see ([21], chapter 3$)$.

Example 50 (see [9]). Let $0<\alpha \leq 1, a=\alpha-1$ and $b$ such that $a \equiv b(\bmod 1)$. Let $y(t)$ be defined on $\mathbb{N}_{a} \cap{ }_{b} \mathbb{N}$. Consider the following Caputo right fractional difference equation:

$$
{ }_{b} \Delta^{\alpha} Q y(t)=\lambda y(2 a+b-t),+f(a+b-t)(Q y)(b)=a_{0} .
$$

If we apply the $Q$ operator on the Caputo fractional difference equation (118), then we obtain the left Caputo fractional difference equation (102). For more information about the $Q$-operator and its use in functional fractional differential equations we refer to [27].

Similar to Example 50 aforementioned, we can use the Q-operator to transform, as well, nabla left type fractional difference equations to right ones and vise versa.

\section{Conclusions}

Two types of dual identities have been used to relate Caputo type fractional differences. The proved dual identities confirm the following.

(1) In the right fractional difference case we most likely need to use both nabla and delta operators.

(2) The (dual) Caputo fractional difference definition is more logical to be used (see Propositions 31 and 32).

\section{Acknowledgments}

The author would like to thank Prince Salman Research and Translation Center in Prince Sultan University for the financial support. This is part of the project (IBRP-OYP-20125-1) supported by Prince Sultan University, Riyadh, KSA, in 2013. 


\section{References}

[1] I. Podlubny, Fractional Differential Equations, vol. 198 of Mathematics in Science and Engineering, Academic Press, San Diego, Calif, USA, 1999.

[2] S. G. Samko, A. A. Kilbas, and O. I. Marichev, Fractional Integrals and Derivatives: Theory and Applications, Gordon and Breach Science, Yverdon, Switzerland, 1993.

[3] A. A. Kilbas, M. H. Srivastava, and J. J. Trujillo, Theory and Application of Fractional Differential Equations, vol. 204 of North Holland Mathematics Studies, Elsevier Science, 2006.

[4] O. P. Agrawal and D. Baleanu, "A Hamiltonian formulation and a direct numerical scheme for fractional optimal control problems," Journal of Vibration and Control, vol. 13, no. 9-10, pp. 1269-1281, 2007.

[5] E. Scalas, "Mixtures of compound Poisson processes as models of tick-by-tick financial data," Chaos, Solitons \& Fractals, vol. 34, no. 1, pp. 33-40, 2007.

[6] D. Baleanu and J. J. Trujillo, "On exact solutions of a class of fractional Euler-Lagrange equations," Nonlinear Dynamics, vol. 52, no. 4, pp. 331-335, 2008.

[7] K. S. Miller and B. Ross, "Fractional difference calculus," in Proceedings of the International Symposium on Univalent Functions, Fractional Calculus, and Their Applications, pp. 139152, Nihon University, Koriyama, Japan, 1989.

[8] H. L. Gray and N. F. Zhang, "On a new definition of the fractional difference," Mathematics of Computation, vol. 50, no. 182, pp. 513-529, 1988

[9] T. Abdeljawad, "On Riemann and Caputo fractional differences," Computers \& Mathematics with Applications, vol. 62, no. 3, pp. 1602-1611, 2011.

[10] F. M. Atıc1 and P. W. Eloe, "A transform method in discrete fractional calculus," International Journal of Difference Equations, vol. 2, no. 2, pp. 165-176, 2007.

[11] F. M. Atıcı and P. W. Eloe, "Initial value problems in discrete fractional calculus," Proceedings of the American Mathematical Society, vol. 137, no. 3, pp. 981-989, 2009.

[12] F. M. Atıcı and P. W. Eloe, "Discrete fractional calculus with the nabla operator," Electronic Journal of Qualitative Theory of Differential Equations, no. 3, pp. 1-12, 2009.

[13] F. M. Atıcı and S. Şengül, "Modelling with fractional difference equations," Journal of Mathematical Analysis and Applications, vol. 369, pp. 1-9, 2010.

[14] N. R. O. Bastos, R. A. C. Ferreira, and D. F. M. Torres, "Discretetime fractional variational problems," Signal Processing, vol. 91, no. 3, pp. 513-524, 2011.

[15] T. Abdeljawad and D. Baleanu, "Fractional differences and integration by parts," Journal of Computational Analysis and Applications, vol. 13, no. 3, pp. 574-582, 2011.

[16] G. A. Anastassiou, "Principles of delta fractional calculus on time scales and inequalities," Mathematical and Computer Modelling, vol. 52, no. 3-4, pp. 556-566, 2010.

[17] G. A. Anastassiou, "Nabla discrete fractional calculus and nabla inequalities," Mathematical and Computer Modelling, vol. 51, no. 5-6, pp. 562-571, 2010.

[18] G. A. Anastassiou, "Foundations of nabla fractional calculus on time scales and inequalities," Computers \& Mathematics with Applications, vol. 59, no. 12, pp. 3750-3762, 2010.

[19] T. Abdeljawad and F. M. Atıc1, "On the definitions of nabla fractional operators," Abstract and Applied Analysis, vol. 2012, Article ID 406757, 13 pages, 2012.
[20] T. Abdeljawad, "Dual identities in fractional difference calculus within Riemann," Advances in Difference Equations, vol. 2013, article 36, 2013.

[21] M. Bohner and A. Peterson, Advances in Dynamic Equations on Time Scales, Birkhäuser, Boston, Mass, USA, 2003.

[22] G. Boros and V. Moll, Irresistible Integrals; Symbolics, Analysis and Experiments in the Evaluation of Integrals, Cambridge University Press, Cambridge, UK, 2004.

[23] R. L. Graham, D. E. Knuth, and O. Patashnik, Concrete Mathematics: Foundation for Computer Science, Addison-Wesley, Reading, Mass, USA, 2nd edition, 1994.

[24] J. Spanier and K. B. Oldham, "The Pochhammer Polynomials $(x)_{n}$," in An Atlas of Functions, pp. 149-156, Hemisphere, Washington, DC, USA, 1987.

[25] F. M. Atıc1 and P. W. Eloe, "Gronwall's inequality on discrete fractional calculus," Computers \& Mathematics with Applications, vol. 64, no. 10, pp. 3193-3200, 2012.

[26] T. Abdeljawad, D. Baleanu, and F. Jarad, "Existence and uniqueness theorem for a class of delay differential equations with left and right Caputo fractional derivatives," Journal of Mathematical Physics, vol. 49, no. 8, article 083507, 11 pages, 2008.

[27] T. Abdeljawad, F. Jarad, and D. Baleanu, "On the existence and the uniqueness theorem for fractional differential equations with bounded delay within Caputo derivatives," Science in China Series A, vol. 51, no. 10, pp. 1775-1786, 2008. 


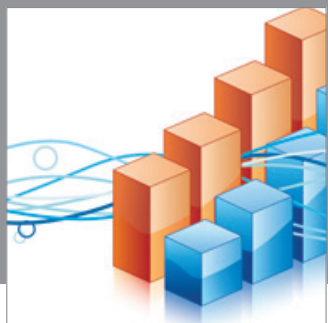

Advances in

Operations Research

mansans

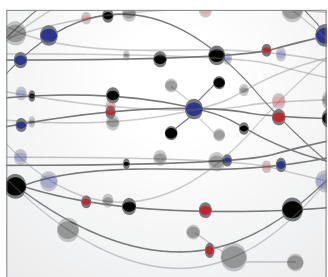

The Scientific World Journal
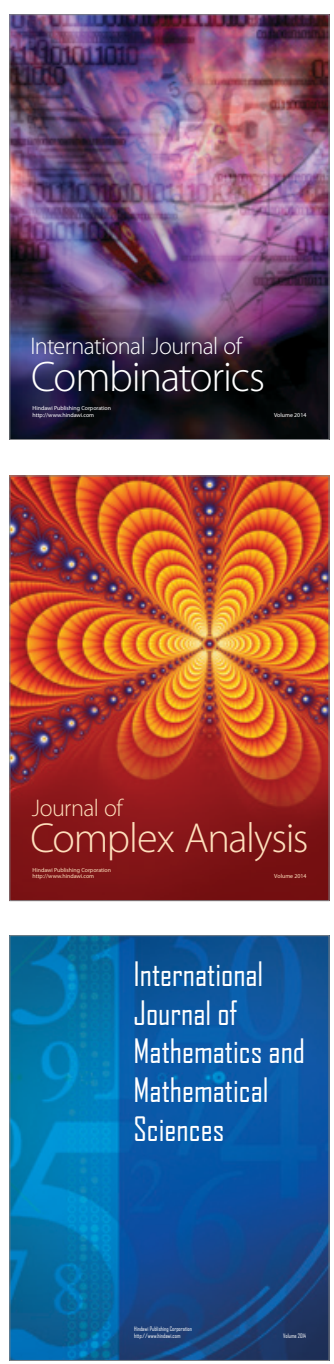
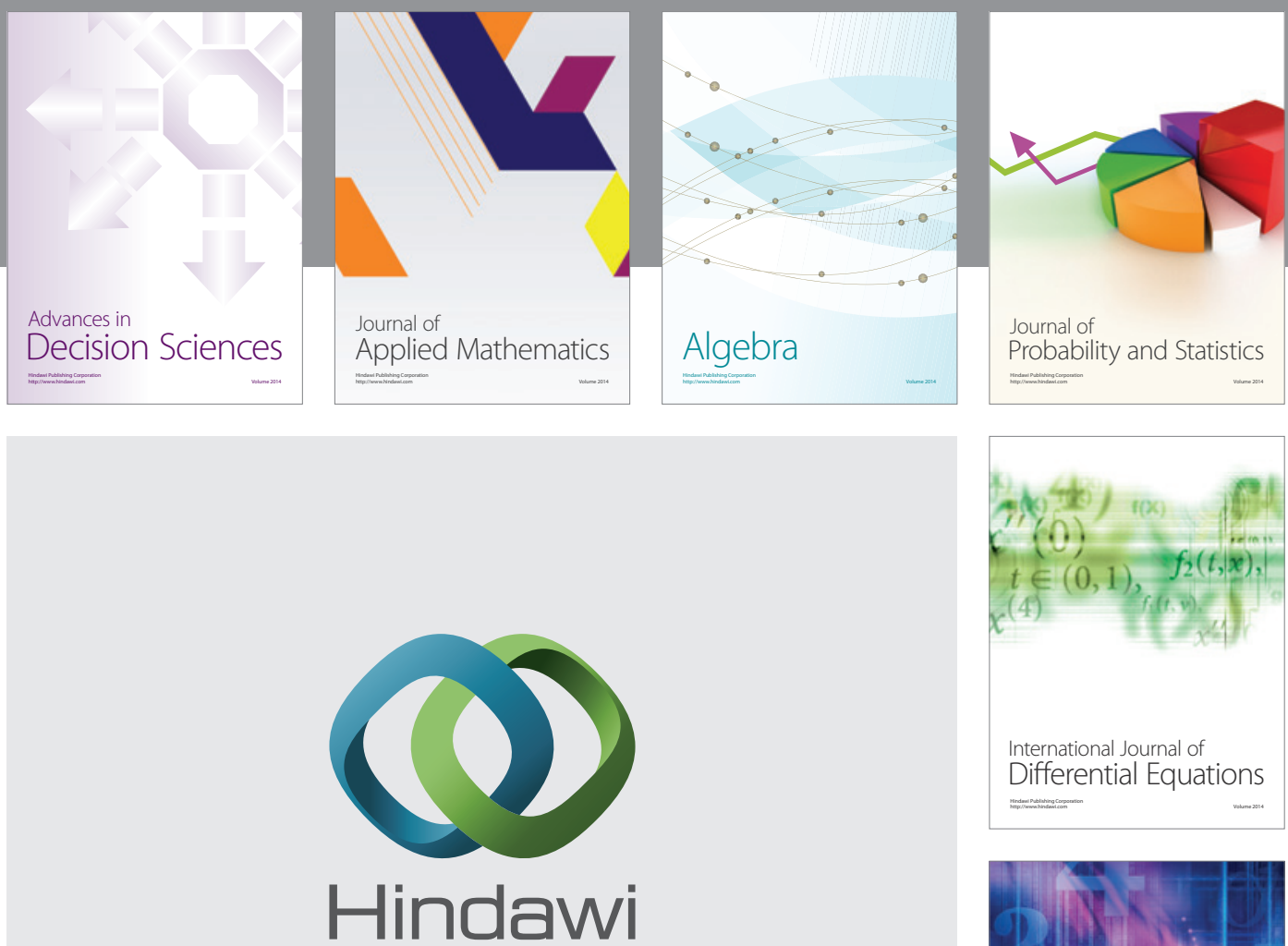

Submit your manuscripts at http://www.hindawi.com
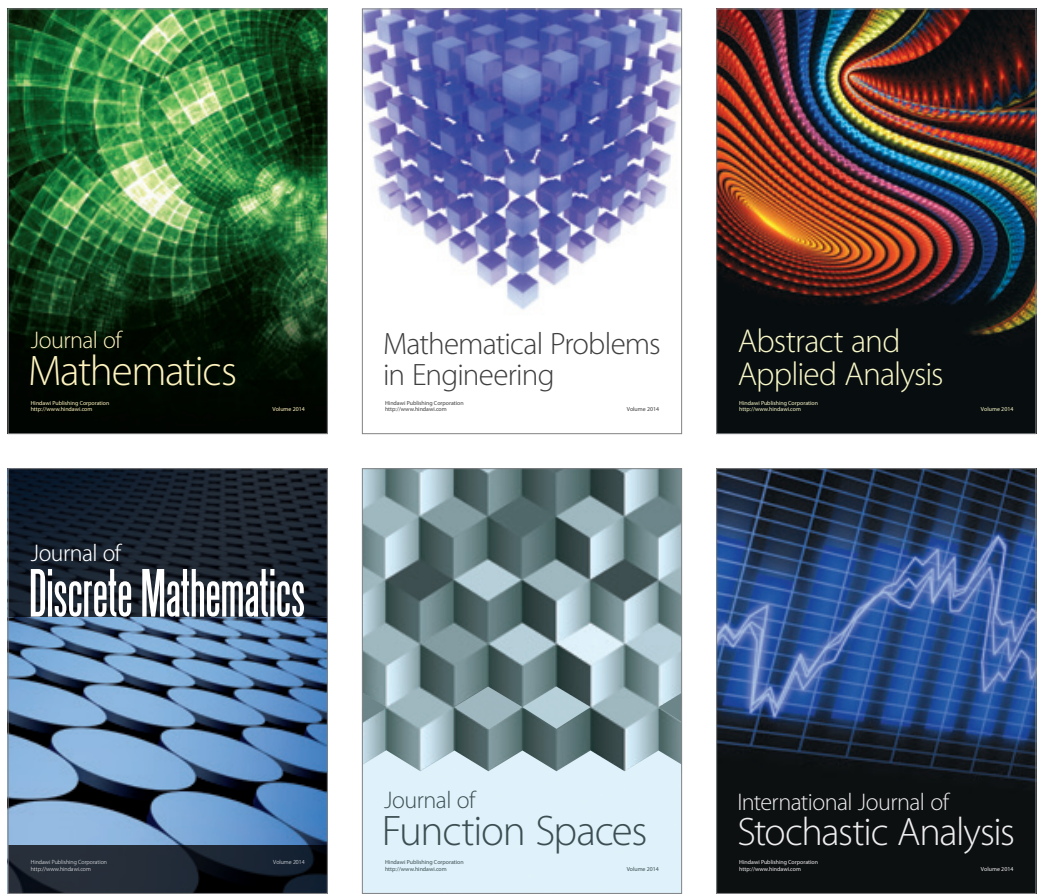

Journal of

Function Spaces

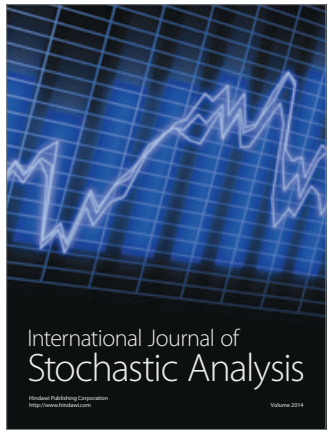

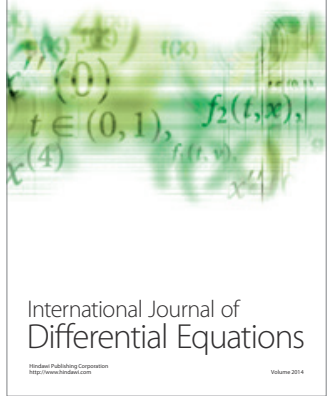
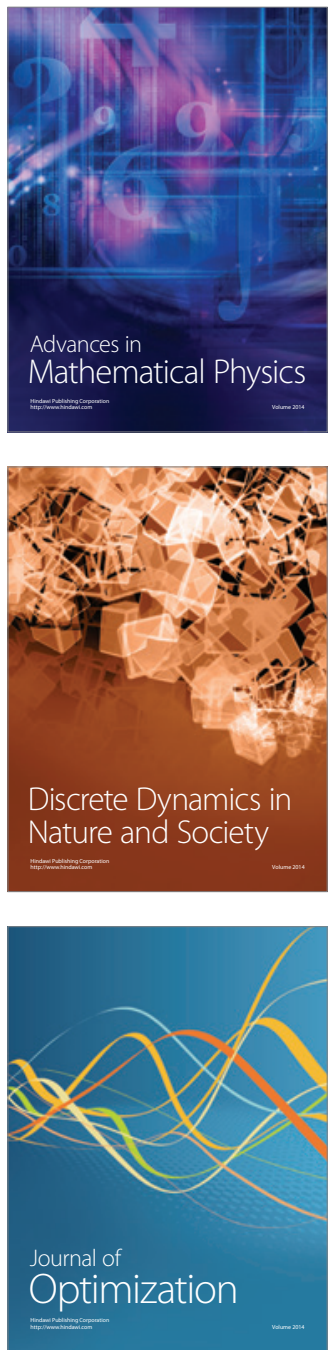